\title{
Clinical Observation on 80 Cases of Anal Edema after Mixed Hemorrhoids Operation with Chinese Medicine Hot Election Package
}

\author{
Yongjiao Zhao, Ximing Zhao \\ Dongzhimen Hospital, Beijing University of Chinese Medicine, Tongzhou Branch, Beijing, China \\ Email: zhaoyongjiao830315@163.com
}

How to cite this paper: Zhao, Y.J. and Zhao, X.M. (2021) Clinical Observation on 80 Cases of Anal Edema after Mixed Hemorrhoids Operation with Chinese Medicine Hot Election Package. Chinese Medicine, 12, 185-195.

https://doi.org/10.4236/cm.2021.123008

Received: August 9, 2021

Accepted: September 11, 2021

Published: September 14, 2021

Copyright $\odot 2021$ by author(s) and Scientific Research Publishing Inc. This work is licensed under the Creative Commons Attribution-NonCommercial International License (CC BY-NC 4.0).

http://creativecommons.org/licenses/by-nc/4.0/

\begin{abstract}
The subjects of this study were 80 patients who underwent mixed hemorrhoids surgery in our hospital from January to October 2020 (mixed hemorrhoids external and internal ligation, internal hemorrhoids ligation or Xiaozhiling injection) and developed anal edge edema after surgery. According to the order of hospitalization, the patients were randomly divided into treatment group and control group, with 40 cases each. In the control group, blank hot pack was applied on the basis of anorectal No. 1 lotion for prevention and treatment of anal edge edema after operation; in the treatment group, hot pack of Traditional Chinese medicine was applied on the basis of anorectal No. 1 lotion for prevention and treatment of anal edge edema. The course of treatment was 7 days. The edema subsidence and postoperative pain scores were observed in both groups. Results: The scores of anal edema and pain in the treatment group were significantly lower than those in the control group $(\mathrm{P}<0.05)$, and the significant efficiency and total effective rate in the treatment group were significantly better than those in the control group, with statistical difference $(\mathrm{P}<0.05)$. Conclusion: Chinese medicine hot election package is effective in treating postoperative anal edema of mixed hemorrhoids, and the method is simple and suitable for clinical promotion.
\end{abstract}

\section{Keywords}

Chinese Medicine Hot Election Package, Mixed Hemorrhoid, Postoperative Anal Edema

\section{Introduction}

Mixed hemorrhoids is a common disease in anorectal surgery, Anal margin edema is the most common complication after mixed hemorrhoids surgery. 
Postoperative anal edge edema of mixed hemorrhoids can cause poor wound drainage, resulting in severe wound pain in patients, and then cause postoperative defecation fear, constipation, defecation efforts aggravate anal edge edema and slow down the speed of wound healing and other chain reactions. The occurrence and progress of edema are rapid, but the problems of slow regression and residual skin excrescences have been troubling the anorectal surgeons. Therefore, how to effectively prevent and treat postoperative invasive edema of mixed hemorrhoids is an urgent problem to be solved by anorectal doctors. I treated the patients admitted to our department from January to October in 2020 who suffered from invasive edema after mixed hemorrhoids surgery, and treated them with traditional Chinese medicine hot election package, which achieved excellent results. The report is as follows.

\section{Data and Methods}

\subsection{General Information}

The subjects were a total of 80 patients who underwent mixed hemorrhoids surgery (external hemorrhoids stripping and internal ligation, internal hemorrhoids ligation or Xiaozhiling injection) in the second district of Anorectal Department, Dongzhimen Hospital, Beijing University of Traditional Chinese Medicine, and developed postoperative peripheral edema. There were 40 patients in the control group, including 18 males and 22 females, with an average age of $45.74 \pm 11.12$ years. There were 40 cases in the treatment group, including 15 males and 25 females, with an average age of $46.75 \pm 11.49$ years old. Statistical analysis showed that there was no significant difference in age and sex between the two groups ( $\mathrm{P}>0.05)$, indicating that the two groups were comparable.

\subsection{Diagnostic Criteria}

1) Mixed hemorrhoid diagnosis criteria for reference Anorectal disease of TCM syndrome diagnostic efficacy of standard [1]: bloody, a mass in the anus, body sensation, belly or pain may be accompanied by the anus, anal partial discharge, or companion has anal local pruritus, anal canal internal tooth line up the same position in the mass (tooth line can also be for the skin), and other clinical symptoms, diagnosis of mixed hemorrhoid.

2) Diagnostic criteria for postoperative hemorrhoid edema: refer to the diagnostic criteria of postoperative hemorrhoid anal edema proposed in the guidelines for the diagnosis and treatment of common diseases in the department of Anal Bowel of Traditional Chinese Medicine in 2012 [2]: a) Hemorrhoid postoperative anal incision tissue raised congestion and edema; b) The symptoms are mainly characterized by swelling and pain, falling and distension, and in severe cases, activities will be limited; c) Thrombophysis was excluded.

\subsection{Inclusion Criteria}

1) In line with the diagnostic criteria of mixed hemorrhoids: anorectal diseases 
of Traditional Chinese medicine diagnosis and efficacy criteria; 2) Between 16 and 70 years old; 3) Postoperative margin edema appeared, and meet the diagnostic criteria of mixed hemorrhoids postoperative anal margin edema; 4) The operation of mixed hemorrhoids stripping and internal hemorrhoids ligation or Xiaozhiling injection; 5) No serious underlying diseases (cardiovascular and cerebrovascular diseases, immune deficiency, severe liver and kidney insufficiency, etc.) and other rectal and anal diseases.

\subsection{Exclusion Criteria}

1) Age $>70$ or $<16$ years old; 2) There is no anal edge edema in the anal wound after mixed hemorrhoids operation; 3) Complicated with serious basic diseases (cardiovascular and cerebrovascular diseases, immune deficiency, severe liver and kidney dysfunction, etc.) and complicated with anal fistula, perianal abscess, anal fissure and other anorectal diseases; 4) Pregnant women, lactation, mental illness; 5) Patients who could not cooperate with the study due to other reasons.

\subsection{Treatment Methods}

Patients in both groups were treated with intraspinal anesthesia for mixed hemorrhoids exudation and internal hemorrhoid ligation or Xiaozhiling injection. After the operation, hemostatic gauze was used to cover the wound, small square gauze was applied for pressure dressing, $\mathrm{H}$ patch was fixed, and supine for 6 hours. In both groups, intravenous antibiotics were used for routine 6 days after surgery to prevent incision infection. Both the treatment group and the control group were given anorectal lotion no. 1 daily from the 1st day after operation (Hedyotis diffusa $30 \mathrm{~g}$, matrine $30 \mathrm{~g}$, Scutellaria baicalensis $20 \mathrm{~g}$, frankincense 15 g, red peony root 20 g, Portulaca oleracea 20 g, Salvia miltiorrhize 20 g, Atractylodes $20 \mathrm{~g}$, Astragalus $20 \mathrm{~g}$, glauber nitrate $20 \mathrm{~g}$ ). This traditional Chinese medicine was made from granules in the Pharmacy of our hospital. The patient poured one bag at a time into the bidet, and mixed it with $1500 \mathrm{ml}$ boiling water to fully dissolve the medicine, after drying it, the patient took a bidet for 15 to 20 minutes, twice a day.

In the treatment group, after fumigation and bathing with anorectal lotion No. 1, the patients were treated by external application of traditional Chinese medicine reelection package. The drug composition was $40 \mathrm{~g}$ glauber's salt particles, $30 \mathrm{~g}$ gypsum particles, $50 \mathrm{~g}$ wine rhubarb particles. Usage: Put the above medicine into the hot bag cloth bag, spray a small amount of water on the surface, put it into the microwave oven, heat it in the microwave oven with medium fire $(70 \%-80 \%)$ for $4 \mathrm{~min}$, then take it out, wait for the temperature of the hot bag to drop to $40^{\circ} \mathrm{C}-45^{\circ} \mathrm{C}$, and apply the anal wound according to the patient's tolerance. The hot compress time should be controlled within 20 - 30 min every day, and take the patient's skin flush and self-feeling comfort as the degree. 2 times/d. Note: Pay attention to the temperature of the hot bag during the treatment process, and it is appropriate to tolerate the skin of the patient, to avoid 
burns, and immediately stop using when the local rash, blisters and other conditions occur.

The control group was treated with blank hot pack after anorectal lotion No. 1 fumigation and sitz bath. The use method of blank hot pack was consistent with that of traditional Chinese medicine hot election package. At the same time, the patient's anal edge edema and postoperative anal pain were closely monitored, and one course of treatment was 7 days.

\section{Clinical Outcome Indicators}

\subsection{Edema of Integral}

After 1 course of treatment, compared two groups of postoperative edema of anal edge points: edema of anal edge criteria: 0 point: without edema; 1 point: mild edema $(<1 / 2$ perianal area); 2 points: moderate edema $(1 / 4-1 / 2$ perianal area); 3 points: severe edema ( $\geq 1 / 2$ perianal area) [3], and patients were scored on the $3 \mathrm{rd}$, 5th and 7th day of treatment, respectively.

\subsection{Postoperative Pain Score}

After 1 course of treatment, postoperative anal pain scores were compared between the two groups: visual analog scale (VAS) was used to score postoperative pain for enrolled patients. In the VAS method, pain is represented by a line segment $10 \mathrm{~cm}$ in length, with numbers representing different pain intensivities. From left to right, they are marked with $0,1,2,3,4,5,6,7,8,9,10.10$ is the most painful and 0 is the painless, and patients mark their most painful point on the back of the line segment. The distance from zero is the score of pain intensity. Then the observation indicators were set into 6 grades according to the score value:

Painless, 0: no obvious feeling of pain;

Mild pain, 2 points: tolerable pain, normal life and sleep;

Moderate pain, 4 points: Pain has slightly affected sleep and requires analgesics.

Moderate to severe pain, 6 points: moderate pain interferes with sleep and requires anesthetic analgesics;

Severe pain, 8 points: sleep disturbance is heavy, accompanied by other symptoms, analgesic drugs effective but not good;

Intolerable, 10 points: seriously affect sleep, accompanied by other symptoms, the patient is often passive posture, anesthesia analgesic drugs effect is not good.

\subsection{Efficacy Criteria}

The percentage change in scores of the two groups was evaluated after 1 course of treatment by the formula: percentage change in scores $=$ (score before administration-score after administration)/score before administration $\times 100 \%$. Significant efficacy: score reduction $\geq 60 \%$; Effective: score reduced by $20 \%-60 \%$; Invalid: Score reduced by $<20 \%[4]$. 


\section{Statistical Methods}

SPSS23.0 statistical software was used to process the data. The counting data were expressed as $[\mathrm{n}(\%)]$ and $\mathrm{X}^{2}$ test was adopted. The measurement data were represented by $(\bar{x} \pm s)$, and their normality and homogeneity of variance were tested. If normal and homogeneity of variance were satisfied, the t-test was used for comparison between groups; if not, the rank sum test was used. Rank sum test was used for grade data, and $\mathrm{P}<0.05$ was considered statistically significant.

\section{Results}

\subsection{Gender Distribution}

There were a total of 80 patients in the two groups, including 40 patients in the treatment group, including 15 males (37.5\%) and 25 females (63.5\%), and 40 patients in the control group, including 18 males (45\%) and 22 females (55\%). The chi-square test $(\mathrm{P}=0.17>0.05)$ showed that there was no statistical difference in gender between the two groups of patients, and the study subjects were comparable (See Table 1 for details).

\subsection{Age Distribution}

The mean age of the control group was $45.74 \pm 11.12$ years, and that of the treatment group was $46.75 \pm 11.49$ years. The $t$-test $(t=-0.325, P=0.756>0.05)$ showed that there was no statistical difference in age distribution between the two groups, and the study subjects were comparable (See Table 2 for details).

\subsection{Comparison of Edema Scores between the Two Groups}

As can be seen from Table 3, the rank sum test results of postoperative edema of mixed hemorrhoids in the two groups showed that $\mathrm{P}=0.697>0.05$ for comparison between the two groups before treatment, showing no statistical difference, indicating that there was no difference in the degree of postoperative anal edema of mixed hemorrhoids in the two groups before treatment after statistical analysis, and the two groups were comparable. 1 day after treatment, the edema score of the two groups was compared $(\mathrm{P}=0.493>0.05)$, showing no statistical difference (suggesting that there was no difference in the treatment of anal edge

Table 1. Gender distribution of patients in the two groups.

\begin{tabular}{cccccc}
\hline Group & Male (\%) & Female (\%) & total & $\mathrm{X}^{2}$ & $\mathrm{P}$ \\
\hline Control group & $18(45)$ & $22(55)$ & 40 & & \\
Treatment group & $15(37.5)$ & $25(63.5)$ & 40 & & 0.197 \\
\hline
\end{tabular}

Table 2. Age distribution of patients in the two groups $(\bar{x} \pm s)$.

\begin{tabular}{ccccc}
\hline Group & Total & age (years) & $\mathrm{t}$ & $\mathrm{P}$ \\
\hline Control group & 40 & $45.74 \pm 11.12$ & & 0.756 \\
Treatment group & 40 & $46.75 \pm 11.49$ & -0.325 & \\
\hline
\end{tabular}


Table 3. Comparison of scores of postoperative anal margin edema in the two groups of patients with mixed hemorrhoids.

\begin{tabular}{cccc}
\hline \multirow{2}{*}{ Group } & \multicolumn{2}{c}{ Edema score } & \multirow{2}{*}{$\mathrm{P}$} \\
\cline { 2 - 3 } & Treatment group $(\bar{x} \pm s)$ & Control group $(\bar{x} \pm s)$ & \\
\hline Before treatment & $2.96 \pm 0.305$ & $2.93 \pm 0.345$ & 0.697 \\
One day after treatment & $2.87 \pm 0.379$ & $2.91 \pm 0.431$ & 0.493 \\
After 5 days of treatment & $1.60 \pm 0.305$ & $2.17 \pm 0.403$ & 0.023 \\
After 7 days of treatment & $0.38 \pm 0.480$ & $1.90 \pm 0.504$ & 0.001 \\
\hline
\end{tabular}

edema between the two groups after ld treatment). After 5 days of treatment, the scores of the two groups for anal rim edema were compared, $\mathrm{P}=0.023<0.05$, the difference was statistically significant. After 7 days of treatment, the scores of the two groups for anal rim edema were compared, $\mathrm{P}=0.001<0.05$, the difference was statistically significant, shows the same time edema scores of treatment group was obviously lower than the control group, it is suggested that traditional Chinese medicine hot election package is better than control group in treating postoperative anal edema of mixed hemorrhoids.

\subsection{Comparison of VAS Scores between the Two Groups after Treatment}

As can be seen from Table 4, the VAS score of the treatment group was significantly better than that of the control group at each time period, and the difference was statistically significant $(P<0.05)$, indicating that the traditional Chinese medicine hot election package was also significantly better than that of the control group in the treatment of anal pain after mixed hemorrhoid surgery. The results are shown in Table 4.

\subsection{Comparison of Post-Treatment Pain Scores between the Two Groups}

It can be seen from Table 5 that after treatment, the score of pain degree in the treatment group was significantly lower than that in the control group, indicating that the traditional Chinese medicine hot election package could significantly reduce the postoperative pain of patients. Compared with the control group, the difference was statistically significant $(\mathrm{P}<0.05)$. The results were shown in Table 5.

\subsection{Treatment Results}

It can be seen from Table 6 that the therapeutic effect evaluation of the two groups showed that the treatment group was superior to the control group in both the significant efficiency and the total effective rate, and the difference was statistically significant after statistical analysis $(\mathrm{P}<0.05)$. In the treatment group, 20 cases of mixed hemorrhoids postoperative anal edge edema were significantly effective, 18 cases were effective, and the effective rate was 95\%. In the control 
Table 4. Comparison of VAS scores between the two groups after treatment.

\begin{tabular}{ccccc}
\hline Time & Treatment group & Control group & $\mathrm{t}$ & $\mathrm{P}$ \\
\hline Day 1 of treatment & $5.90 \pm 0.92^{*}$ & $6.83 \pm 1.01$ & -6.17 & 0.0105 \\
The 5th day of treatment & $2.76 \pm 0.93^{*}$ & $4.59 \pm 1.08$ & -3.39 & 0.0002 \\
Day 7 of treatment & $1.64 \pm 0.45^{*}$ & $2.83 \pm 0.38$ & -2.21 & 0.0008 \\
\hline
\end{tabular}

Note: Comparison between treatment group and control group ${ }^{\star} \mathrm{P}<0.05$.

Table 5. Comparison of pain scores between the two groups after treatment.

\begin{tabular}{ccccc}
\hline Time & Treatment group & Control group & $\mathrm{t}$ & $\mathrm{P}$ \\
\hline Day 1 of treatment & $5.45 \pm 1.30^{*}$ & $6.95 \pm 1.23$ & -6.14 & 0.0123 \\
The 5th day of treatment & $3.05 \pm 1.77^{\star}$ & $4.15 \pm 1.86$ & -3.25 & 0.0029 \\
Day 7 of treatment & $1.88 \pm 1.67^{\star}$ & $3.01 \pm 1.29$ & -2.22 & 0.0000 \\
\hline
\end{tabular}

Note: Comparison between treatment group and control group ${ }^{\star} \mathrm{P}<0.05$.

Table 6. Comparison of efficacy evaluation between the two groups (N) \%.

\begin{tabular}{cccccc}
\hline Group & N & Showed effective & Effective & Ineffective & Total response rate/\% \\
\hline Control group & 40 & 8 & 22 & 10 & 75.00 \\
Treatment group & 40 & 20 & 18 & 2 & 95.00 \\
$\mathrm{X}^{2}$ & & & & & 8.193 \\
$\mathrm{P}$ & & & & & 0.002 \\
\hline
\end{tabular}

group, 8 cases of mixed hemorrhoids with anal edge edema after operation were shown to be effective by external application of blank hot election package, and 22 cases were effective. The effective rate was $75 \%$. By $\mathrm{X}^{2}$ test, $\mathrm{P}=0.002<0.05$, there was a significant difference between the two groups. See Table 6 for details.

\section{Discussion and Conclusion}

The most common disease in anorectal surgery is mixed hemorrhoids. If mixed hemorrhoids are ineffective after conservative treatment, surgical treatment is required. Although mixed hemorrhoids surgery is very small, there are many postoperative complications, among which postoperative anal edge edema is the most common complication after mixed hemorrhoids surgery, mainly shown as edge of wound or skin bridge congestion and edema, swelling and pain, which seriously delayed the postoperative wound healing speed of patients. It aggravated the postoperative pain and increased hospitalization time and hospitalization cost of the patients. There are many reasons for anal edge edema after mixed hemorrhoids surgery, the most important are the following points: 1) surgical operation and internal hemorrhoids ligation will directly block or stasis of the local meridian of the anus qi, qi stagnation will lead to blood stasis, blood stasis operation is not smooth, obstruction will lead to pain, so it will cause local 
anal swelling, pain and discomfort, anal edge edema. 2) Wound pain after mixed hemorrhoids surgery will cause spasm of the internal sphincter, and then cause obstruction of the reflux of the inferior rectal vein and lymph circulation, increased vascular permeability, tissue fluid accumulation and increased exudation of inflammatory substances, leading to local edema [5]. 3) After mixed hemorrhoids surgery, because the patient's defecation receptors are damaged, the anus will fall and swell and the stool will not feel good. Squatting or defecation time is too long or the stool is dry, which can increase the pressure of the anal canal, the venous congestion around the anus, affect the blood lymphatic reflux, and cause the anal incision edema [6]. Generally speaking, the TCM pathogenesis of anal edge edema after mixed hemorrhoids operation is dampness and heat injection, qi stagnation and blood stasis, and meridian block. After mixed hemorrhoids operation, the author applied anorectal No. 1 lotion fumigation and washing sitz bath combined with Chinese medicine hot election package to treat anal edge edema, clear heat, dampness and detoxification, promote blood circulation, remove stasis, reduce swelling and relieve pain, the effect is remarkable.

Chinese medicine hot election package is an important part of Chinese medicine external treatment. In ancient Chinese medicine books, there has long been the view of "external treatment and transdermal drug delivery". Such as the general Record of Shengii stated: the external treatment should be carried out from the outside to the inside, and the paste, ironing and steaming bath powder can be used to reach the qi. As is known to all, the human body is rich in capillaries, Due to its warm and hot nature, Chinese medicine reelection package can warm the meridians, promote blood circulation and remove blood stasis. It can increase the temperature of local meridians and acupoints applied by the heat package, local temperature can accelerate the local blood circulation. The skin penetrates deep into the tissue and penetrates from the outside to the inside layers to give full play to the efficacy of Chinese medicine ingredients in a higher quality [7].

In this study, the efficacy of traditional Chinese medicine hot election package is mainly to promote blood circulation, remove blood stasis, anti-inflammatory, sterilization, detumorization and pain relief. Its main components are: $40 \mathrm{~g}$ glauber's salt particles, $30 \mathrm{~g}$ gypsum particles, $50 \mathrm{~g}$ wine rhubarb particles. Glauber's salt began to record in the Mingyi Bielu, It is a mineral traditional Chinese medicine, cold in nature and salty and bitter in taste. its main composition is $\mathrm{Na}_{2} \mathrm{SO}_{4} \cdot 10 \mathrm{H}_{2} \mathrm{O}$, which has the effect of clearing away heat, detoxifying, relieving inflammation and relieving pain. External application to the affected area can clear away heat, soft and firm to eliminate carbcarbous swelling, and significantly inhibit the swelling caused by early inflammatory exudate [8]. Glauber's salt gels in local can spur local capillary expansion, accelerate the blood flow velocity and tiny circulation, make the site form hypertonic environment, so as to make the tissue fluid can seep quickly, can very good play the role of rapid elimination of local tissue edema, moreover also can accelerate the local lymph circulation, reduce the local inflammatory cell infiltration, and then reduce the local in- 
flammatory response and play an anti-inflammatory effect. Modern pharmacological studies have proved that mircanite crystal has obvious stimulating effect on reticuloendothelial system, enhancing the system's hyperplasia and phagocytic function, and then reducing inflammatory edema; after absorption, mirabilite can stimulate the vagus nerve, cause local small blood vessels to dilate, improve local blood circulation significantly, accelerate local lymphatic production, and effectively promote the dissipation of edema [9]. Rhubarb is bitter in taste and cold in nature. It can purge fire and detoxify, promote blood circulation and remove blood stasis, and purge constipation. Ancient Chinese people used rhubarb in external treatment very early, for example, the "Pinhu Jijianfang" said: "To treat the wounds of servants ... rhubarb external apply, one night black will turn purple, and two nights purple will turn white." Modern pharmacological studies have found that rhubarb can inhibit bacterial growth and reproduction by inhibiting bacterial biological oxidase system. It plays an anti-inflammatory role by inhibiting the synthesis of leukotriene B4 by human polymorphonuclear leukocytes. At the same time, by increasing the fibrinogen and platelet content, promoting blood coagulation to achieve hemostasis, so it has broad-spectrum anti-inflammatory, antibacterial and hemostatic effects [10]. Modern pharmacological studies also found that rhubarb through emodin and other active components to change capillary permeability, remove inflammatory substances, reduce fluid extravasation into the interstitial space, and play the role of hemostasis, blood circulation, anti-inflammatory, antibacterial and other effects; Tannic acid, the main component of rhubarb, can play an astringent effect by inhibiting secretion, and has anti-infection effect, which can inhibit most gram-positive bacteria and some gram-negative bacteria [11]. Therefore, the combination of rhubarb and glauber's nitrate can promote blood circulation of the affected area, accelerate the convergence of local water in the edema, inhibit the growth of bacteria on the wound, reduce swelling and stop pain, significantly promote the dissipation of edema, and accelerate the healing of the tissue. Gypsum is spicy and slightly cold, clear and relieves heat, reduce swelling and relieves pain. It is recorded in "Bencao Jing Jie" that the external use of gypsum can clear and relieve heat and poison, reduce swelling and relieve pain to treat gold sore. Ling Yunpeng once said: "gypsum is cold ... And the formation of ulcers, mostly caused by heat and meat decay, with the distribution of the part, help to local anti-inflammatory and detumify. Modern pharmacological studies have found that gypsum has a strong and rapid antipyretic effect, and it is also effective in regulating the metabolic disorders of trace elements caused by pathological changes and enhancing the bactericidal immunity of the body [12]. Yang Liu et al. found that gypsum can achieve antipyretic and anti-inflammatory effects by regulating PGE2 content [13]. The above three kinds of traditional Chinese medicine are combined in reasonable doses to promote blood circulation and remove blood stasis, reduce swelling and relieve pain, and have the effects of anti-inflammatory and sterilization.

The author first uses anorectal No. 1 lotion to fume and wash the sez bath, 
and uses the heat of steam and the medicinal power of Traditional Chinese medicine to directly act on the perianal skin and cou reason, so that the local skin pores of perianal skin open small blood vessels to expand, so that the skin and wound tissue directly absorb the effective components of traditional Chinese medicine, but also can make the local anal sphincter relax capillary dilation, Thus further promoting perianal local lymphatic reflux and blood circulation, thus effectively promoting the dissipation of peripheral edema [14]. After the traditional Chinese medicine fumigation and zit bath, the treatment of the traditional Chinese medicine hot election package is more conducive to the absorption of the active ingredients of the hot election package by the skin and wound tissue, so as to effectively promote the dissipation of the wound edema, and also avoid the first pass effect of the drug in the liver, so as to reduce the toxicity and adverse reactions. To sum up, the treatment of mixed hemorrhoids with traditional Chinese medicine hot election package has a significant effect on postoperative anal edge edema, and the clinical operation of this method is simple, and it is worthy of widespread use by anorectal doctors in clinical practice.

\section{Conflicts of Interest}

The authors declare no conflicts of interest regarding the publication of this paper.

\section{References}

[1] National Administration of Traditional Chinese Medicine (1995) Criteria for Diagnosis and Curative Effect of Anorectal Diseases in Traditional Chinese Medicine. Nanjing University Press, Nanjing, 131.

[2] China Association of Traditional Chinese Medicine (2012) Guidelines for Diagnosis and Treatment of Common Diseases in Anorectal Department of Traditional Chinese Medicine. China Press of Traditional Chinese Medicine, Beijing, 10-13.

[3] Yu, B.M., Lin, J.J., Wu, W.Q., et al. (2004) Treatment of Acute Hemorrhoids with Purified Flavonoid Compounds: A Multi-Center Self-Controlled Clinical Study. Chinese Journal of General Surgery, 19, 722.

[4] Wang, Y., Fang, X.C. and Chen, J. (2010) Clinical Observation of Maizhilin Tablet in Improving Postoperative Anal Edge Edema of Hemorrhoids. Practical Clinical Integrated Traditional Chinese and Western Medicine, L0, 60.

[5] Jiang, W., Zhang, H.X., Sui, N., et al. (2016) Epidemiological Survey of Common Anorectal Diseases in Chinese Urban Residents. Chinese Journal of Public Health, 32, 1293-1296.

[6] Zhang, A. (2017) Analysis and Management of Postoperative Anal Margin Edema of Mixed Hemorrhoids. Chinese Journal of Modern Medicine, 17, 155-158.

[7] Xing, S.X., HAO, G.T., Ren, X.L., et al. (2014) Therapeutic Effect of Sodium Hyaluronate on Knee Osteoarthritis. World Journal of Integrated Chinese and Western Medicine, 9, 975-977.

[8] Yana, Z.H. (2009) Chinese Medicine. People's Health Press, Beijing, 297-305.

[9] Yang, J.X. and Bi, H.X. (1998) Pharmacodynamics of Traditional Chinese Medicine. China Press of Traditional Chinese Medicine, Beijing, 249-250. 
[10] Song, H.Y., Zou, X.M., Luo, Q., et al. (2010) Application of Rhubarb and Mirabilite Combined External Application in Obstetric Perineotomy. International Journal of Nursing, 29, 1882-1884.

[11] Mine Load Right (.2001) Chinese Materia Medica. 2nd Edition, Shanghai Science and Technology Publishing House, Shanghai, 98-99.

[12] Sun, S. (2009) Study on Pharmacological Action and Trace Elements of Gypsum. Modern Distance Education of Traditional Chinese Medicine, 7, 170.

[13] Yang, L., Zhang, Y.S., et al. (2016) Research Progress on Antipyretic Effect of Mineral Gypsum. Chinese Journal of Medicine, 19, 1943-1945. (in Chinese with English Abstract)

[14] Guo, Q.L., He, Q.F. and Luo, L.H. (2013) Treatment of Postoperative Mixed Hemorrhoids by Traditional Chinese Medicine. Journal of Integrative Medicine, 8 , 1280-1283. 